\title{
El riesgo cardiovascular basal es útil para la toma de decisiones en el tratamiento antihipertensivo
}

Usefulness of baseline cardiovascular risk to guide blood pressure-lowering treatment decisions

\section{Objetivos}

Determinar si los beneficios de los antihipertensivos son proporcionales al riesgo cardiovascular basal para establecer si el riesgo absoluto puede utilizarse en la toma de decisiones terapéuticas.

\section{Fuentes de datos}

En esta revisión sistemática y meta-análisis se incluyeron 11 ensayos clínicos aleatorizados (ECA), con un total de 67.475 individuos.

\section{Selección de estudios}

Para ser elegibles, los estudios debían haber aleatorizado a los pacientes o bien a una droga activa vs. placebo, o bien comparar tratamientos más o menos intensivos. Asimismo, debían tener un mínimo de 1.000 pacientes-año de seguimiento planificado por rama y no haber presentado sus resultados antes de la finalización del protocolo, en Julio de 1995.

\section{Extracción de datos}

El punto final principal fue un combinado de eventos cardiovas-
Sundström J y col. Lancet 2014;384:591-8. culares mayores (accidente cerebrovascular, enfermedad coronaria, insuficiencia cardiaca o mortalidad cardiovascular). Se estratificó a los participantes en cuatro niveles de riesgo absoluto a cinco años, sobre la base de co-variables y eventos tales como edad, sexo, índice de masa corporal, presión arterial (PA) sistólica y diastólica, tabaquismo, diabetes y antecedentes de enfermedad cardiovascular, desarrollando para ello ecuaciones de riesgo para cada uno de los puntos finales individuales. Se determinó la reducción del riesgo para descensos de $5 \mathrm{mmHg}$ y $5 \%$ de la PA sistólica basal.

\section{Resultados Principales}

Los antihipertensivos se asociaron a un beneficio relativo similar a lo largo de diferentes estratos de riesgo cardiovascular. El beneficio absoluto del tratamiento se incrementó a medida que se incrementaba el riesgo basal, previniendo progresivamente más eventos cardiovasculares y disminuyendo el número necesario a tratar (NNT) de sujetos para prevenir un evento (Tabla 1).

Tabla 1: Efecto de la reducción de presión arterial sobre el riesgo relativo y absoluto de enfermedad cardiovascular por grupo de pacientes con diferente riesgo basal.

\begin{tabular}{c|c|c|c|c}
\hline Riesgo a 5 años de ECV & Riesgo relativo (IC95\%) & RRA (IC95\%) & NNT & $\begin{array}{c}\text { Diferencia promedio de PA } \\
\text { sistólica/diastólica (mmHg) }\end{array}$ \\
\hline Menor a 11\% & $0,82(0,73$ a 0,93) & $-1,41(-2,05$ a $-0,77)$ & 71 & $4,6 / 3,0$ \\
\hline 11 a 15\% & $0,85(0,75$ a 0,96$)$ & $-1,95(-3,09 \mathrm{a}-0,82)$ & 51 & $6,0 / 3,2$ \\
\hline 15 a 21\% & $0,87(0,78$ a 0,98$)$ & $-2,41(-4,04 \mathrm{a}-0,77)$ & 41 & $7,1 / 3,2$ \\
\hline Mayor a 21\% & $0,85(0,76$ a 0,95$)$ & $-3,84(-6,06 \mathrm{a}-1,61)$ & 26 & $5,9 / 3,0$ \\
\hline
\end{tabular}

ECV: enfermedad cardiovascular. IC95\%: intervalo de confianza del 95\%. NNT: número necesario a tratar. PA: presión arterial. RRA: reducción del riesgo absoluto. *A favor del tratamiento activo.

\section{Conclusiones}

El descenso de la PA implica una protección relativa similar a medida que se incrementa el riesgo basal. Sin embargo, las reducciones son progresivamente crecientes respecto del riesgo absoluto. Estos resultados avalan el uso de ecuaciones de riesgo cardiovascular basal para guiar las decisiones respecto del tratamiento antihipertensivo.
Fuente de financiamiento/conflicto de interés de los autores: $\mathrm{El}$ estudio no recibió financiamiento. Los autores declaran conflictos de interés respecto de las siguientes empresas: Itrim, Novartis, Dr Reddy's Laboratories, AbbVie, AstraZeneca, Baxter, Boehringer Ingelheim, Servier, Vitae, Janssen, Roche, Abbott, Pfizer.

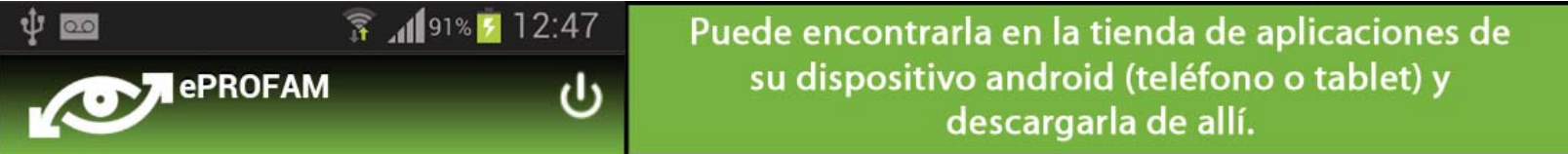

\section{NUEVA APLICACIÓN PARA DISPOSITIVOS ELECTRÓNICOS}

Desarrollada por el Servicio de Medicina Familiar y Comunitaria del Hospital Italiano de Buenos Aires, Argentina.
ePROFAM es un resumen actualizado de terapéutica en medicina ambulatoria y constituye una herramienta práctica y accesible para que el médico utilice en el consultorio frente al paciente. 


\section{Comentario}

Desde hace tiempo es tema de controversia qué criterios utilizar para tomar decisiones respecto del inicio o ajuste del tratamiento antihipertensivo. Las guías de hipertensión europeas del $2007^{1}$ proponían evaluar integralmente al paciente, no sólo a través su valor de PA, sino teniendo en cuenta su riesgo cardiovascular global. Así, algunos pacientes que no llegaban a valores de 140 y/ó 90 podrían requerir tratamiento. Sin embargo, sus autores debieron dar marcha atrás, reconociendo que la evidencia para dicha recomendación no era contundente, postura que se ha mantenido en la actualización de las guías del $2013^{2}$. En el presente meta-análisis se retoma esta cuestión, y si bien sus conclusiones parecerían apoyar el uso del riesgo basal por sobre el valor de tos importantes: 1) si bien se incluyeron miles de pacientes, las ecuaciones desarrolladas para este meta-análisis carecen de validez externa, lo que limita su generalización; 2) no se reportan efectos adversos del tratamiento por estrato de riesgo; 3 ) el promedio de PA era elevado en todos los grupos de riesgo, desde el más bajo $(155 / 94 \mathrm{mmHg})$ hasta PA para el cambio de conducta, deben tenerse en cuenta ciertos aspec-

el más alto (165/88 mmHg), con lo cual no hay respuesta respecto de si tratar o no a pacientes con PA limítrofe pero que son de alto riesgo por otros factores; 4) la PA fue evaluada con escasas mediciones en consultorio, de conocida menor precisión que los métodos para evaluar la PA ambulatoria ${ }^{3}$; 5) los ECA incluidos son previos a 1995, por lo que los tratamientos de otros factores de riesgo pueden no haber estado acordes a los estándares actuales, sesgando los resultados.

\section{Conclusiones del comentador}

La evaluación integral del paciente para guiar las decisiones terapéuticas, más allá del valor de PA, resulta atractiva y es crucial la generación de evidencia al respecto. Actualmente no contamos con una herramienta universal para dicha evaluación así como tampoco con aval suficiente para instituir tratamiento en algunos pacientes de alto riesgo con valores limítrofes de PA, ni tampoco para negárselo a aquellos con valores de PA elevado y bajo riesgo de acuerdo a otros factores.

Jessica Barochiner [ Sección Hipertensión Arterial, Servicio de Clínica Médica del Hospital Italiano de Buenos Aires. jessica.barochiner@hospitalitaliano.org.ar]

Barochiner J. El riesgo cardiovascular basal es útil para la toma de decisiones en el tratamiento antihipertensivo. Evid Act Pract Ambul. 2016;19(1):1011. Ene-Mar. Comentado de: Blood Pressure Lowering Treatment Trialists, y col. Blood pressure-lowering treatment based on cardiovascular risk: a meta-analysis of individual patient data. Lancet. 2014;384. PMID: 25131978.

\section{Referencias bibliográficas}

1. Mancia G, y col. ESH-ESC Task Force on the Management of Arterial Hypertension. 2007 ESH-ESC Practice Guidelines for the Management of Arterial Hypertension: ESHESC Task Force on the Management of Arterial Hypertension. J Hypertens. 2007;25(9):1751-62.

2. Mancia G,y col. Task Force for the Management of Arterial Hypertension of the European Society of Hypertension and the European Society of Cardiology. 2013 ESH/ESC Practice Guidelines for the Management of Arterial Hypertension. Blood Press. 2014;23(1):3-16.

3. Parati G, y col. European Society of Hypertension Working Group on Blood Pressure Monitoring and Cardiovascular Variability. European Society of Hypertension practice guidelines for ambulatory blood pressure monitoring. J Hypertens. 2014;32(7):1359-66.

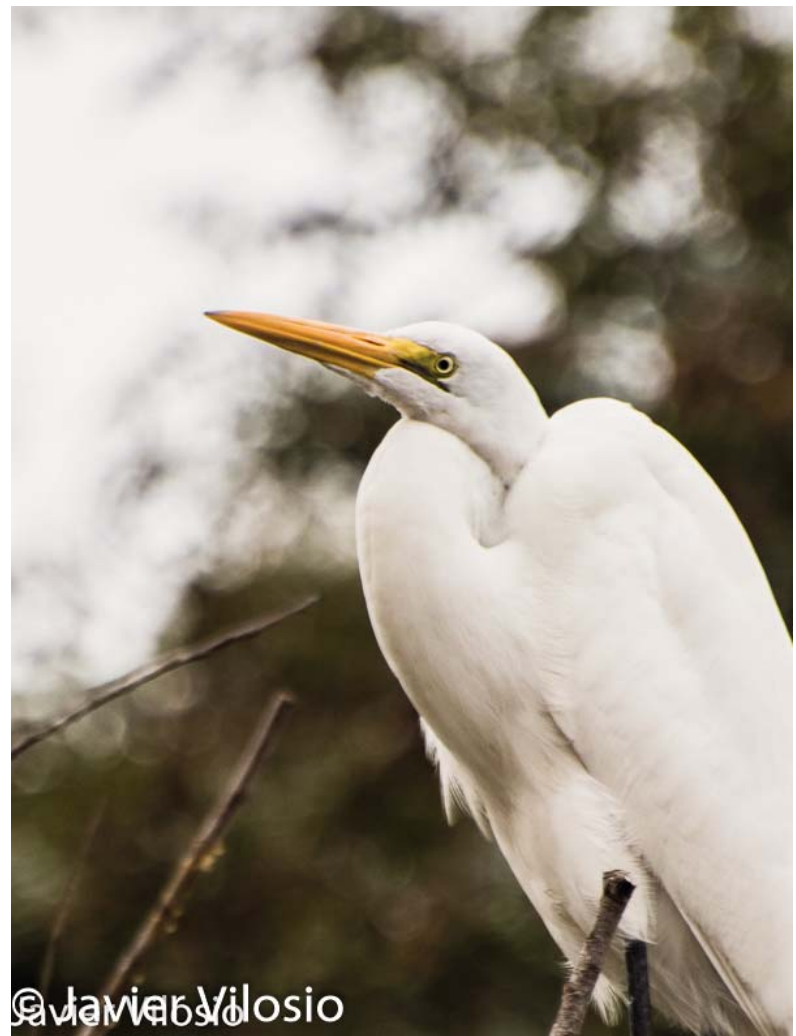

Fotografía: Javier Vilosio 